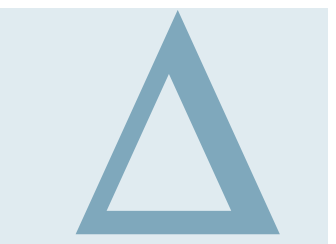

CHANGE PAGE

\title{
Using a combination inhaler (budesonide plus formoterol) as rescue therapy improves asthma control
}

\author{
Peter J Barnes
}

PJ Barnes, Airway Disease Section, National Heart and Lung Institute, Imperial College, London

p.j.barnes@imperial.ac.uk

BMJ 2007;335:513

do: 10.1136/bmj.39273.537778.AD

References, a methods box, and table showing results of the supporting trials are on bmj.com
Change Page aims to alert clinicians to the immediate need for a change in practice to make it consistent with current evidence. The change must be implementable and must offer therapeutic or diagnostic advantage for a reasonably common clinical problem. Compelling and robust evidence must underpin the proposal for change.

Series adviser: Joe Collier (changepage@bmj.com), emeritus professor of medicines policy, St George's, University of London.

\section{The clinical problem}

Asthma is effectively controlled in most patients with maintenance treatment. In those with moderate or severe persistent asthma, control may be achieved with an inhaled corticosteroid or a combination inhaler containing a corticosteroid and a long acting $\beta_{2}$ agonist. The combination inhaler is more effective, but patients still require short acting $\beta_{2}$ agonists such as salbutamol or terbutaline to relieve symptoms. I describe a new approach for acute exacerbation-SMART (single inhaler maintenance and reliever therapy). This uses the combination inhaler, rather than the short acting $\beta_{2}$ agonist, as the reliever.

\section{The evidence for change}

Using a formulation of budesonide plus formoterol (budesonide/formoterol) both as a reliever and as maintenance therapy once or twice daily is more effective in controlling asthma than conventional approaches using budesonide/formoterol, fluticasone/salmeterol, or high dose corticosteroids (budesonide or fluticasone) as the maintenance treatment with short acting $\beta_{2}$ agonists as relievers (table). ${ }^{1-5}$ The most striking benefits are fewer exacerbations (severe exacerbations, defined as needing a course of oral corticosteroids, are about halved) and fewer admissions to hospital. The mean number of extra puffs of budesonide/formoterol taken in the SMART studies was about one a day. The single inhaler approach with budesonide/formoterol seems to be effective in mild, moderate, and severe persistent asthma.

Previous studies have shown that inhaled formoterol is even more effective than salbutamol as a rescue therapy, with an onset as fast and with similar side effects. ${ }^{6}$ However, using the budesonide/formoterol inhaler as rescue therapy is more effective than formoterol alone, indicating that the inhaled corticosteroid in the rescue inhaler also plays an important role. ${ }^{7}$

Analysis of asthma exacerbations shows a slow evolution over several days with increasing symptoms and use of rescue medication. ${ }^{8}$ Studies in unselected populations also show that, as an exacerbation develops, patients increase the dose of rescue inhalers but not usually the dose of inhaled corticosteroid. ${ }^{9}$ This new, simplified, single inhaler approach may also improve compliance.

The fluticasone/salmeterol combination inhaler is unsuitable as a rescue therapy as salmeterol has a slower onset of action than formoterol and cumulative side effects. However, other combination inhalers (with different corticosteroids plus formoterol) are now in development; a beclomethasone/formoterol inhaler is already available in Germany.

\section{KEY POINTS \\ Single inhaler therapy for asthma with combined budesonide and formoterol as maintenance therapy and also as a reliever provides better control of asthma than combination inhalers or higher doses of inhaled corticosteroids as maintenance plus a short acting $\beta 2$ agonist as reliever \\ The greatest benefit is the reduction of exacerbations \\ The strategy is simple for patients to adopt \\ Overuse of rescue combination therapy does not seem to be a problem}

\section{Barriers to change}

Early experience suggests that it is helpful for patients to have two combination inhalers: one at home for maintenance therapy, the other carried for rescue therapy. An initial concern was that patients may overuse the rescue inhaler and therefore end up taking high doses of inhaled corticosteroids. This practice was not seen in the large clinical trials, presumably as the SMART strategy was more effective in controlling asthma. Patients should be advised to see their doctor if they need to use more than six additional puffs of budesonide/formoterol rescue inhaler.

Another concern is the cost of the SMART approach. On average, however, only one extra inhalation of combination therapy daily is used, so there is little increase in the total drug cost, whereas the reduction in severe exacerbations results in overall healthcare saving. ${ }^{10}$ Also, as the new inhalers containing a corticosteroid plus formoterol come on to the market, the drug costs are likely to drop. Because controlled clinical trials include selected patients, studies of clinical effectiveness in unselected populations are now needed.

\section{How should we change our practice?}

Single inhaler therapy using combined budesonide/formoterol for both maintenance and rescue therapy is more effective than conventional therapy. Eligible patients are those with moderate to severe asthma whose condition is not controlled with conventional therapy and who are using rescue inhaler therapy.

Single inhaler maintenance and reliever therapy is easy to implement as the patient merely replaces their usual rescue inhaler with the budesonide/formoterol inhaler. Maintenance budesonide/formoterol may be given twice daily for severe asthma (or once daily for moderate asthma). ${ }^{2}$ The strategy has also been shown to be effective in children aged 4-12 years but has not yet been approved by regulatory authorities for use in them. ${ }^{11}$ Single inhaler therapy is not suitable for patients who overuse their rescue inhalers or who find it difficult to recognise if their asthma is worsening. Competing interests: None declared. 